\title{
Profile of Clinical and Radiological Factors of Intracerebral Hemorrhage Stroke Patients in Dr. Soetomo General Hospital Surabaya
}

\author{
Farizal Rizky Muharram¹, Asra Al Fauzi ${ }^{2}$, Paulus Rahardjo ${ }^{3}$, Pudji Lestari ${ }^{4}$
}

\author{
${ }^{1}$ Faculty of Medicine, Universitas Airlangga, Surabaya. \\ 2 Department of Neurosurgery, Faculty of Medicine, Universitas Airlangga, Surabaya. \\ ${ }^{3}$ Department of Radiology, Faculty of Medicine, Universitas Airlangga, Surabaya. \\ ${ }^{4}$ Department of Public Health and Preventive, Faculty of Medicine, Universitas Airlangga, Surabaya.
}

\section{A B S T R A C T}

Introduction: Intracerebral hemorrhage $(\mathrm{ICH})$ is a medical emergency with high mortality and morbidity that occurs in $15-20 \%$ of stroke cases. However, in the Multicenter Surgical Trial in Lobar ICH (STICH) study no benefit was found from the initial operative handler in Lobar ICH patients. Many clinical and radiological factors affect the outcomes of patients. This study aims to find variables that have significance in the mortality rate of $\mathrm{ICH}$ stroke patients

Methods: The design of this study was a cross-sectional study. Clinical and radiological factors were analyzed by chi-square test and Kruskal-Wallis test. Then significant results were sought Relative Risk. This research was conducted from January 2017 to September 2018 starting from sampling to processing data. Sampling was carried out at the medical record center of Dr. Soetomo General Hospital Surabaya. Data collection is done by using secondary data in the form of patient medical records

Results: Age $(p=0.0014)$, Glasgow Comma Scale $(p=0.000)$, Tentorial location $(p=0.034)$, Intraventricular hemorrhage $(p=0.000)$, Hemorrhage volume $(p=0.024)$, $\mathrm{ICH}$ Score $(p=000)$ had significant role that predict outcome of patient with $\mathrm{ICH}$. But Sex $(p=0.937)$ and Treatment $(p=0.796)$ didn't have significant role.

Conclusion: There are several factors that influence the outcome of $\mathrm{ICH}$ patients namely: Age, Glasgow Coma Scale, Hemorrhage Location, Intraventricular Hemorrhage, Volume of Hemorrhage, and $\mathrm{ICH}$ Score. Radiological factors and clinical factors both have an impact on outcomes.

* Correspondence: asraalfauzi@gmail.com

JUXTA: Jurnal IImiah Mahasiswa Kedokteran Universitas Airlangga

p-ISSN: 1907-3623; e-ISSN: 2684-9453

DOI: 10.20473/juxta.V10I12019.15-19

Open access under Creative Commons Attribution-ShareAlike 4.0 International License (CC-BY-SA)
ARTICLE INFO

Article history:

Received 04 December 2018

Received in revised form 22 January 2019

Accepted 24 January 2019

\section{Keywords:}

$\mathrm{ICH}$,

Radiological Factors,

Clinical Factors,

Operative,

Conservative. 


\section{Introduction}

Intracerebral hemorrhage $(\mathrm{ICH})$ can be treated with medical conservative treatment, surgical operative, and minimally invasive surgery. At present, there is no adequate treatment for $\mathrm{ICH}$. Several studies have tried to compare outcomes from the operative and conservative methods. One of the most famous studies is the study of Surgical Treatment in Lobar Intracranial Haemorrhage (STICH). The STICH study was conducted by the Multicenter in 1995. The STICH study found no benefit from early operative handlers in Lobar ICH patients ${ }^{1-3}$.

In the STICH study, there are a number of issues that are criticized by clinicians where the time of handling is not divided, the depth or volume of uncharged $\mathrm{ICH}$ makes it biased towards the treatment of patients with operative surgery. With more advanced CT-Scan and better evaluation of Radiodiagnosis factors, STICH II is currently being carried out in response to the criterion of STICH 14,5

Based on the STICH research data, it can be concluded that operative measures provide benefits for $\mathrm{ICH}$ with Infratentorial locations. But for the Supratentorial location, there is still debate and controversy for handling operatives ${ }^{6}$. Here the author wants to try to examine more specifically which subgroups benefit from operative Treatments by comparing clinical factors, namely the level of consciousness (Glasgow Coma Scale) and age ${ }^{7}$. As well as radiological factors, namely the volume of bleeding ${ }^{8}$, the location of bleeding ${ }^{2}$, and intraventricular expansion ${ }^{9}$.

This study aims to find variables that have signed on the mortality rate of $\mathrm{ICH}$ stroke patients. So that in the future there will be a more appropriate treatment for certain characteristics. which is expected to reduce mortality.

\section{Methods}

The design of this study was a cross-sectional study with a retrospective observational approach to determine the outcome based on the relationship of the radiological and clinical characteristics of stroke patients with the criteria for inclusion of patients in neurology and neurosurgery at the Dr. Soetomo General Hospital Surabaya. The patient has a primary $\mathrm{ICH}$. And the exclusion of patients with: Status of medical records is incomplete, in this case, the clinical and radiological characteristics studied are not recorded. The patient has not received therapy or has received therapy for less than 3 months. Patients experience other vascular disorders, such as aneurysms. The minimum required sample is calculated using the formula Charan and Biswas ${ }^{10}$, included $p$-value according to the lowest mortality in the previous study, namely $20 \% Z=1.96$ and $d=0.05$ the minimum sampling was 240 . Then clinical and radiological factors were analyzed by test chi-square and KruskalWallis test. Then significant results are sought for Relative Risk

\section{Results}

This research was conducted from January 2017 to September 2018 starting from sampling to processing data. Sampling was carried out at the medical record center of Dr. Soetomo General Hospital Surabaya. Data collection is done by using secondary data in the form of patient medical records. All of the collected intracerebral hemorrhage patients were 302 and the total patients who met the inclusion and exclusion criteria were included in the study as the study subjects. Patients who met the criteria obtained 247 research subjects. In the output, variables are divided into two categories, life, and death categories. It can be seen that the percentage of respondents in the living category was $53.8 \%$ with a frequency of 133 respondents. Meanwhile, the death category is $46.2 \%$ with the frequency of respondents being 114 people.

\section{Age}

Age variables are divided into five categories, namely categories of children, young adults, adults, the elderly, and very elderly. It can be seen that the percentage of respondents in the child category is $2.0 \%$ with a frequency of 5 respondents. Young adult category is $20.2 \%$ with a frequency of 50 people. Adult category is $62.3 \%$ with a frequency of 154 respondents. The elderly category is $14.2 \%$ with a frequency of 35 . Meanwhile, the percentage of the category is very elderly at $1.2 \%$ with a frequency of 3 respondents. Then performed a statistical test and obtained $p=0.014$ and $t=-2.47$ were obtained. then the conclusion of the age ratio is inversely proportional to the outcome of the patient's life. The older the patient the higher the death rate (See at table 1).

\section{Sex}

In gender, variables are divided into two categories, namely categories of men and women. It can be seen that the percentage of male patients is $54.7 \%$ (135 respondents) and the percentage of female patients is $45.3 \%$ (112 respondents). Then performed a statistical test and obtained $p$-value $=0.937$. Which means there is no significant difference in the outcome of patients in different Genders.

\section{Glasgow Coma Scale (GCS)}

GCS variables were divided into three categories, namely the conscious category $(>13)$, unconscious (5-13) and unconscious $(<5)$. It can be seen that the percentage of GCS respondents in the conscious category $(0-4)$ is $28.3 \%$ with a frequency of 70 respondents. The unconscious category is $58.7 \%$ with a frequency of 145 respondents. Meanwhile, the percentage of unconscious categories (13-15) is $13 \%$ with a frequency of 32 respondents. Then performed a statistical test and obtained $p$-value $=0.000$. Which means there are significant differences in the outcome of patients in different GCs. In 
the trend, there was a decrease in GCS followed by an increase in mortality.

\section{Tentorial location}

In Tentorial Location variables are divided into two categories, namely the Supratentorial and Infratentorial categories. It can be seen that the percentage of respondents in the Tentorial location in the supratentorial category was $90.3 \%$ with a frequency of 223 respondents. Meanwhile, the infratentorial category was $9.7 \%$ with a frequency of 24 respondents. Then a statistical test is performed and $p$-value $=0.034$ is obtained. Which means there are significant differences in the outcome of patients on different Tentorial Locations. In the trend seen Supratentorial had a lower mortality rate compared to infratentorial patients ( $41 \%$ vs $33 \%)$.

\section{Intraventricular Hemorrhage (IVH)}

The IVH variables are divided into two categories, namely the categories IVH and Non-IVH. It can be seen that the percentage of IVH respondents in the Non-IVH category was $48.2 \%$ with a frequency of 119 respondents. Meanwhile, the IVH category was $51.8 \%$ with a frequency of 128 respondents. Then a statistical test is performed and $\mathrm{p}$-value $=0.000$ is obtained. Which means there are significant differences in patient outcomes in patients with IVH. In the trend seen IVH patients had higher mortality rates compared to no-IVH patients $(71 \%$ vs $38 \%)$.

\section{Hemorrhage Volume}

Volume variables are divided into three categories, namely low, medium, and high categories. It can be seen that the percentage of respondents in the low category is $55.1 \%$ with a frequency of 136 respondents. Meanwhile, the Medium category was $31.6 \%$ with a frequency of 78 respondents. In the high category, the percentage of respondents was $13.4 \%$ with a frequency of 33 respondentsThen performed a statistical test and obtained $p$-value $=0.024$. Which means there are significant differences in the outcome of patients in different volumes. The trend seen as higher volume will be followed by an increase in mortality.

\section{ICH Score}

In $\mathrm{ICH}$ Score variables are divided into seven categories, namely categories zero to six. It can be seen that the percentage of respondents to the $\mathrm{ICH}$ Score in the zero categories is $12.6 \%$ with a frequency of 31 respondents. The first category is $20.6 \%$ with a frequency of 51 respondents. Category two is $31.2 \%$ with the frequency of respondents 77 people. Category three is $23.5 \%$ with the frequency of respondents 58 people. The fourth category is $9.7 \%$ with a frequency of 24 people. Category five is $2.0 \%$ with a frequency of 5 people.

Table 1. Descriptive and Significance Test
Meanwhile, category six is $4 \%$ with a frequency of respondents of 4 people.Then performed a statistical test and obtained $p$-value $\alpha=0,000$. Which means there are significant differences in patient outcomes on different $\mathrm{ICH}$ scores. In the trend seen an increase in $\mathrm{ICH}$ Score is directly proportional to the increase in mortality.

\section{Hemorrhage Location}

The location variables are divided into seven categories, namely the basal ganglia, lobar, thalamus, cerebellum, multiple, brainstem, and other categories. It can be seen that the percentage of location respondents in other categories is $2.0 \%$ with a frequency of 5 respondents. The basal ganglia category was $33.2 \%$ with a frequency of 82 respondents. The lobar category is $32.8 \%$ with a frequency of 81 respondents. The thalamus category is $12.6 \%$ with the frequency of respondents 31 people. The cerebellum category was $4.9 \%$ with a frequency of 12 respondents. Multiple categories of $9.3 \%$ with a frequency of 23 respondents. Meanwhile, the brainstem category was $5.3 \%$ with a frequency of respondents of 13 people.Then performed a statistical test and obtained $p$-value $\alpha=0.009$. Which there are significant differences in the outcome of the patient in patients with different bleeding sites.

\section{Treatments}

The Treatment variables are divided into two categories, namely conservative and operative categories. It can be seen that the percentage of respondent Treatments in the conservative category amounted to $57.9 \%$ with a frequency of 143 respondents. Meanwhile, the operative category was $42.1 \%$ with a frequency of 104 respondents. Then performed a statistical test and obtained $p$-value $=0.796$. Which means there is no significant difference in the outcome of the patient in operative and conservative treatment. Both conservative and operative Treatments which are not subgroups have a different mortality rate.

\section{Discussion}

After analyzing each variable on the outcome of $\mathrm{ICH}$ patients. then the writer analyzes statistical tests to answer how each factor influences outcomes. the results of our study found that the age ratio is inversely proportional to the outcome of the patient's life $(P=0.0014)$. The older the patient the higher the death rate. This is in line with the study of Rendevski et al, Independently, age affects the outcome of patients significantly at a young age with a higher percentage of life ${ }^{6}$. This is because in young adult patients the entire physiology of the body is still running well and the regeneration power is still high. So that the healing power of major surgery is better ${ }^{7,11}$.

The results of our study which showed that GCS obtained $p$-value $=0.000$. Which means there are significant differences in the outcome of patients in 


\begin{tabular}{|c|c|c|c|c|c|c|c|}
\hline No & Variable & Category & Life & Death & Total & $\begin{array}{l}\text { Mortality } \\
\text { Percentage }\end{array}$ & P-Value \\
\hline \multirow{6}{*}{1} & \multirow{6}{*}{ Age } & Child (0-20) & 3 & 2 & 5 & $40 \%$ & \multirow{6}{*}{0.014} \\
\hline & & Young Adult (20-40) & 33 & 17 & 50 & $34 \%$ & \\
\hline & & Adult $(40-60)$ & 79 & 75 & 154 & $49 \%$ & \\
\hline & & Elderly $(60-80)$ & 17 & 18 & 35 & $51 \%$ & \\
\hline & & Very Elderly (>80) & 1 & 2 & 3 & $67 \%$ & \\
\hline & & Total & 133 & 114 & 247 & $46 \%$ & \\
\hline \multirow{3}{*}{2} & \multirow{3}{*}{ Sex } & Male & 73 & 62 & 135 & $46 \%$ & \multirow{3}{*}{0.937} \\
\hline & & Female & 60 & 52 & 112 & $46 \%$ & \\
\hline & & Total & 133 & 114 & 247 & $46 \%$ & \\
\hline \multirow{4}{*}{3} & \multirow{4}{*}{ GCS } & $>13$ & 56 & 14 & 70 & $20 \%$ & \multirow{4}{*}{0.000} \\
\hline & & $5-13$ & 70 & 75 & 145 & $52 \%$ & \\
\hline & & $<5$ & 7 & 25 & 32 & $78 \%$ & \\
\hline & & Total & 133 & 114 & 247 & $46 \%$ & \\
\hline \multirow{3}{*}{4} & \multirow{3}{*}{$\begin{array}{l}\text { Tentorial } \\
\text { Location }\end{array}$} & Supratentorial & 125 & 98 & 223 & $44 \%$ & \multirow{3}{*}{0.034} \\
\hline & & Infratentorial & 8 & 16 & 24 & $67 \%$ & \\
\hline & & Total & 133 & 114 & 247 & $46 \%$ & \\
\hline \multirow{3}{*}{5} & \multirow{3}{*}{ IVH } & Non-IVH & 85 & 34 & 119 & $29 \%$ & \multirow{3}{*}{0.000} \\
\hline & & IVH & 48 & 80 & 128 & $63 \%$ & \\
\hline & & Total & 133 & 114 & 247 & $46 \%$ & \\
\hline \multirow{4}{*}{6} & \multirow{4}{*}{ Volume } & $<30 \mathrm{cc}$ & 81 & 55 & 136 & $40 \%$ & \multirow{4}{*}{0.024} \\
\hline & & $30-60 \mathrm{cc}$ & 41 & 37 & 78 & $47 \%$ & \\
\hline & & $>60 \mathrm{cc}$ & 11 & 22 & 33 & $67 \%$ & \\
\hline & & Total & 133 & 114 & 247 & $46 \%$ & \\
\hline \multirow{8}{*}{7} & \multirow{8}{*}{$\mathrm{ICH}$ Score } & 0 & 28 & 3 & 31 & $10 \%$ & \multirow{8}{*}{0.000} \\
\hline & & 1 & 37 & 14 & 51 & $27 \%$ & \\
\hline & & 2 & 40 & 37 & 77 & $48 \%$ & \\
\hline & & 3 & 21 & 37 & 58 & $64 \%$ & \\
\hline & & 4 & 6 & 18 & 24 & $75 \%$ & \\
\hline & & 5 & 1 & 4 & 5 & $80 \%$ & \\
\hline & & 6 & 0 & 1 & 1 & $100 \%$ & \\
\hline & & Total & 133 & 114 & 247 & $46 \%$ & \\
\hline \multirow{8}{*}{8} & \multirow{8}{*}{ Locations } & Others & 3 & 2 & 5 & $40 \%$ & \multirow{8}{*}{0.009} \\
\hline & & Basal Ganglia & 50 & 32 & 82 & $39 \%$ & \\
\hline & & Lobar & 49 & 32 & 81 & $40 \%$ & \\
\hline & & Thalamus & 9 & 22 & 31 & $71 \%$ & \\
\hline & & Cerebellum & 5 & 7 & 12 & $58 \%$ & \\
\hline & & Multiple & 14 & 9 & 23 & $39 \%$ & \\
\hline & & Brainstem & 3 & 10 & 13 & $77 \%$ & \\
\hline & & Total & 133 & 114 & 247 & $46 \%$ & \\
\hline & & Conservative & 78 & 65 & 143 & $45 \%$ & \\
\hline 9 & Treatment & Operative & 55 & 49 & 104 & $47 \%$ & 0.796 \\
\hline & & Total & 133 & 114 & 247 & $46 \%$ & \\
\hline
\end{tabular}

different GCs. In the trend, there was a decrease in GCS followed by an increase in mortality. This is in line with a previous study of GCS by Castellanos et al 2005 and Davis et al $2006{ }^{12,13}$

The results of our study that showed an increase in volume increasing the mortality rate of $\mathrm{ICH}$ patients with significant results $(P=0.024)$. This is in line with a previous study of radiological factors by Castellanos et al 2005 and Davis et al $2006^{12,13}$, which showed that bleeding volume> $30 \mathrm{cc}$ and hematoma expansion would significantly increase patient mortality. This is due to the high volume of bleeding increasing the ICP which causes herniation of Cerebri' ${ }^{12-14}$. Radiological factors Tentorial sites also show Infratentorial locations have higher mortality rates with significant results $(P=0.034)$.
Whereas, on radiological factors, IVH showed ventricular extension increasing intraventricular mortality with significant results $(P=0.00)$.

All points assessed in the $\mathrm{ICH}$ Score show the significance of the outcome of the death rate. This is in accordance with the research of Hemphill, et al., $2001^{15}$ regarding the $\mathrm{ICH}$ Score which showed an increase in the $\mathrm{ICH}$ score significantly increasing patient mortality $(\mathrm{P}=$ $0.000)^{15}$.

The statistical tests on each location specifically which showed significant results $(P=0.009)$ with the highest mortality rates in the highest order were Brainstem, multiple, Thalamus, Cerebellum, Lobar and Basal Ganglia. The results of this study are not much different from the results of previous studies which showed that based on the location of bleeding, the hospital mortality rate in $\mathrm{ICH}$ stroke patients was $16.3 \%$ in the basal ganglia / internal capsule, 
$20 \%$ in the cerebellum, $25 \%$ in lobar, $25,8 \%$ in the thalamus, $40 \%$ in the brain stem, and $44.4 \%$ in intraventricular ${ }^{14,16}$

Further research is needed to find out which factors can be a diagnosis for choosing treatments that provide better outcomes. Further analysis is needed to look for indications of the use of Operative or Conservative actions.

\section{Conclusion}

There are several factors that influence the outcome of $\mathrm{ICH}$ patients namely: Age, Glasgow Coma Scale, Hemorrhage Location, Intraventricular Hemorrhage, Volume of Hemorrhage, and $\mathrm{ICH}$ Score. Radiological factors and clinical factors both have an impact on outcomes. Statistically, more than 1 variable is needed to determine an action that results in a more significant outcome of living patients. This shows that the choice of treatment for patients with different characteristics requires different treatments.

\section{CONFLICT OF INTEREST}

The author stated there is no conflict of interest.

\section{REFERENCES}

1. Kim H-T, Lee J-M, Koh E-J and Choi H-Y. Surgery versus Conservative Treatment for Spontaneous Supratentorial Intracerebral Hemorrhage in Spot Sign Positive Patients. Journal of Korean Neurosurgical Society. 2015; 58: 30915.

2. Aguilar MI and Brott TG. Update in Intracerebral Hemorrhage. The Neurohospitalist. 2011; 1: 148-59.

3. de Oliveira Manoel AL, Goffi A, Zampieri FG, et al. The Critical Care Management of Spontaneous Intracranial Hemorrhage: A Contemporary Review. Critical Care (London, England). 2016; 20: 272-

4. Mendelow AD, Gregson BA, Mitchell PM, et al. Surgical Trial in Lobar Intracerebral Haemorrhage (STICH II) Protocol. Trials. 2011; 12: 124-.
5. Ikram MA, Wieberdink RG and Koudstaal PJ. International Epidemiology of Intracerebral Hemorrhage. Current Atherosclerosis Reports. 2012; 14: 300-6.

6. Rendevski V, Stojanov D, Aleksovski B, et al. Surgical Outcome in Patients with Spontaneous Supratentorial Intracerebral Hemorrhage. 2017.

7. Chung J-Y, Chang W-Y, Lin T-W, et al. An Analysis of Surgical Outcomes in Patients Aged 80 years and Older. Acta Anaesthesiologica Taiwanica. 2014; 52: 153-8.

8. Broderick JP, Brott TG, Duldner JE, Tomsick T and Huster G. Volume of Intracerebral Hemorrhage. A Powerful and Easy-to-Use Predictor of 30-day Mortality. Stroke. 1993; 24: 987-93.

9. Hanley DF. Intraventricular Hemorrhage: Severity Factor and Treatment Target in Spontaneous Intracerebral Hemorrhage. Stroke. 2009; 40: 1533-8.

10. Charan J and Biswas T. How to Calculate Sample Size for Different Study Designs in Medical Research? Indian Journal of Psychological Medicine. 2013; 35: 121-6.

11. A AK and Valson JS. Surgery in Spontaneous Intracerebral Hemorrhage- A Series Analysis. Journal of Neurology and Stroke. 2015; 2.

12. Rodríguez-Yáñez M, Castellanos M, Freijo MM, et al. Clinical Practice Guidelines in Intracerebral Haemorrhage. Neurología (English Edition). 2013; 28: 236-49.

13. Davis SM, Broderick J, Hennerici M, et al. Hematoma Growth is A Determinant of Mortality and Poor Outcome After Intracerebral Hemorrhage. Neurology. 2006; 66: 1175-81.

14. Salihović D, Smajlović D and Ibrahimagić OĆ. Does the Volume and Localization of Intracerebral Hematoma Affect Short-Term Prognosis of Patients with Intracerebral Hemorrhage? ISRN Neuroscience. 2013; 2013: 327968-.

15. Hemphill JC, Greenberg Steven M, Anderson Craig S, et al. Guidelines for the Management of Spontaneous Intracerebral Hemorrhage. Stroke. 2015; 46: 2032-60.

16. Arboix A, Comes E, García-Eroles L, et al. Site of Bleeding and Early Outcome in Primary Intracerebral Hemorrhage. Acta Neurologica Scandinavica. 2002; 105: $282-8$. 